\title{
Puerperal Peritonitis Treated by Abdominal Section: Notes of Ten Consecutive Cases.
}

\author{
By A. Knyvett Gondon, M.B. (Cantab.), \\ Medical Superintendent of Monsall Fever Hospital, Manchester; \\ Lecturer on Infectious Diseases in the University of Manchester.
}

THE following cases have arisen consecutively in my practice at Monsall Hospital, and it has occurred to me that they may be worth reporting very briefly. I have purposely very much condensed the notes of each case, so that the salient features only are recorded.

For the original notes $I$ am to a great extent indebted to the careful work of the Assistant Medical Officers, Doctors Reid, Hodge, Arnold, Dawson, Wilkins, Linton, and Pell-Ilderton. For the performance of the operations I am personally responsible.

CaSe I. E.S., aged 44, multipara, was admitted to Monsall Hospital on October 3rd, 1905.

The history in this case is very unsatisfactory. The patient was attended by a locum tenens, whose term of engagement expired on the day following the notification of the case, and it was not possible to obtain any details beyond the facts that the patient had been confined on September 27th, and that the forceps had been used.

When I saw her on her admission, she was unconscious and apparently moribund. The temperature was $100.8^{\circ}$, and the radial pulse could not be felt. The cardiac rate was 184 . The abdomen was enormously distended and immobile. The perineum was lacerated centrally down to but not into the rectum, and the cervix was torn diagonally into the vagina.

Laparotomy was at once performed, and the abdominal cavity was found to be full of fetid pus; the intestines were greatly distended, and covered with lymph; the uterus was much enlarged and flabby. From the patent end of each Fallopian tube pus was seen to be exuding. No attempt was made to cleanse the peritoneal cavity. Both tubes were ligatured, and a free opening was made into the posterior cul-de-sac, through which a large rubber and gauze drain was inserted, a similar drain being placed in the lower angle of the abdominal wound. Saline infusions with adrenalin were given into the connective-tissue under the breasts. The patient rallied somewhat, became conscious, and expressed herself as comfortable and free from pain, but she gradually sank, and died twelve hours after the operation. 
Post mortem, the uterus was found to contain sloughing placental masses. Cultures from the peritoneal pus and from the uterus showed streptococci only. Those made from the blood in the basilic vein during life gave no growth.

CASE II. E.B., aged 24, multipara, was admitted to Monsall Hospital on October 24th, 1905.

She had been confined three weeks previously in the presence of a midwife only. The labour is stated to have been easy, but not precipitous. She got up on the sixth day, and was apparently fairly well for three weeks, when she consulted a medical man for pain in the back.

With the history of parturition in mind, he dilated the cervix with Hegar's dilators; no anæsthetic was used, and the cervix was not steadied with volsellæ, no assistance being available. Almost immediately afterwards the patient felt faint, and on the next day she was seriously ill; the temperature was raised, but to what extent is not stated. She became steadily worse, and was admitted three days later.

When I saw her on admission she was obviously very ill; the temperature was $101^{\circ}$, and the pulse-rate was 112 ; she complained of severe pain over the whole of the abdomen; there was no distension, and nothing to be felt on palpation.

On further examination, under chloroform, the cervix was seen to be torn diagonally downwards and to the left; the deeper part of this wound extended behind the uterus into both broad ligaments, but as no attempt was made to determine the upper limit of the laceration (the surfaces being lightly adherent) it was uncertain whether the peritoneal cavity had been opened up or not; the body of the uterus was about $3 \frac{1}{2}$ inches long.

The cavity resulting from the laceration was lightly packed with gauze, and the vagina was then irrigated. The general condition improved somewhat, and the temperature fell, reaching the normal three days later. On October 30 th it rose again, and the patient vomited and complained of increased abdominal pain. On the next day she was markedly worse, and on November 1st there was some rigidity of the abdominal wall with slight distension. I then opened the abdomen.

On the right side the Fallopian tube was distended to the size of a sausage, with pus. Near its fimbriated extremity was a hole, through which some pus had escaped into the peritoneal cavity, giving rise to a condition of general peritonitis with turbid effusion. The visceral peritoneum was markedly injected and covered in places with lymph. The intestines were uniformly distended.

There was a similar pyosalpinx in the situation of the left tube, and an abscess in the right ovary, neither of which communicated 
with the peritoneal cavity. Behind the uterus, in the middle line, was an abscess, equal in size to a large walnut, shut off by adhesions from the general peritoneal cavity, but communicating with the vaginal laceration below.

The general peritoneal cavity was neither flushed nor sponged out; both tubes and the right ovary were removed, and the abscess behind the uterus was drained into the vagina; a large rubber tube was placed in the posterior cul-de-sac, which was freely opened, and a Keith's tube inserted through the abdominal wound.

With the exception of a sharp attack of bronchitis which occurred on November 17th, and in which the patient nearly lost her life, the recovery was satisfactory, and she left the hospital on January $25 \mathrm{th}$, 1906, well and strong, and with both wounds healed.

Cultures from the peritoneal fluid showed both streptococci and bacilli coli communes.

Case Ir. B.B., aged 37, nullipara, was admitted to Monsall Hospital on January 13th, 1906.

The history is, that she had had a miscarriage on January 3rd, had remained in bed for two days, and was at work the next day. She did not consult a medical man until January 13th, when she was at once sent to hospital. Accurate information of the onset of the illness could not be obtained, but it appeared that she was not markedly ill until January 11th, when she was seized with headache, vomiting and shivering.

On admission, her condition was exceedingly grave; the temperature was $103.5^{\circ}$, and the pulse-rate 160 . There was considerable distension of the abdomen, which was immobile, and there was shifting dulness in each flank. Per vaginam there was bulging effusion into Douglas's pouch. The uterus was fixed.

An opening was first made into the posterior cul-de-sac, and much pus evacuated. It was then found that all the pelvic organs were matted together by adhesions, which could not be satisfactorily dealt with by the vaginal route, so the abdomen was opened forthwith. It was then seen that the peritonitis was general; the intestines were everywhere distended and covered with patches of sticky lymph, and about a pint and a half of pus was evacuated from the general abdominal cavity. Both Fallopian tubes were greatly thickened and inflamed, and pus was exuding through the patent ends. The left ovary was simply a shell containing pus; there was a small abscess in the right ovary also.

Both Fallopian tubes were ligatured and removed; the left ovary was removed completely, and the abscess in the right ovary was excised and its bed stitched up, a portion of healthy ovarian tissue being left. No attempt was made to flush the abdominal cavity, which was drained through the posterior vaginal incision, and also 
by a gauze and rubber drain inserted into the abdominal wound which was then closed.

On the next day, the patient was markedly better, and her recovery was subsequently uneventful. She left hospital on April 3rd well and strong.

The leucocyte count before the operation was 22,000. From the peritoneal pus, gonococci and streptococci were found.

It is probable that a condition of chronic gonorrhoea existed before pregnancy in this case, and that the streptococcal infection was due to events which determined the abortion.

CASE IV. R.II., primipara, aged 27, was admitted to Monsall Hospital on February 18th, 1906.

She had been confined on February 6th, the labour being easy and of short duration. A medical man was present, but he did not find it necessary to use the forceps, or to introduce his hand into the uterus. The exact date of the onset of the attack of septicæmia which followed is uncertain, inasmuch as the patient was suffering from "bronchitis" at the time of delivery, and felt more or less ill immediately afterwards. On February 13th, however, there was pyrexia, the temperature remaining high from that day until her admission. During that period there were also abdominal pain, sweating and diarrhœa, and continuous vomiting from February 15 th. Vaginal douches were given, but no other local treatment was adopted.

On admission, the patient appeared to be moribund. The temperature was $103.5^{\circ}$, and the pulse could not be felt at the wrist. The cardiac rate was 196 . The face was pale, and the lips were blue. The abdomen was greatly distended and immobile. Per vaginam, a collection of fluid could be felt behind the uterus, apparently free in the peritoneal cavity; the broad ligaments were greatly thickened.

The abdomen was opened by a long median incision, and a condition of general peritonitis was seen. About three pints of pus were evacuated by turning the patient on to her side. The source of the infection was found to be the left Fallopian tube, from the patent ostium of which pus was exuding. This was ligatured, and free drainage was established through an opening in the posterior cul-desac. A tube was also inserted in the lower angle of the abdominal wound. No attempt was made to cleanse the peritoneal cavity. Five pints of saline solution with ten minims of adrenalin were injected under the breasts, but the patient never rallied, and died eight hours after the operation.

A post mortem examination was, unfortunately, not obtainable, but it is probable that the uterus contained some decomposing 
products of conception. Cultures taken from the blood during life showed streptococci only, while those from the uterus gave streptococci, bacilli coli communes and staphylococci.

Case v. M.J.H., multipara, aged 23, was admitted to Monsall Hospital on February 23rd, 1906.

The history of this case is very indefinite, inasmuch as the patient was not attended in her confinement by either a medical man or a midwife. She was delivered, however, on February 19th, and the labour was probably easy and of short duration. She felt ill on February 20th, and was seen by the certifying practitioner on February 21st. There was then pyrexia, and on the following day there were repeated rigors, abdominal pain, sweating and diarrhoa.

When I saw her on admission, she was apparently moribund. The temperature was $103^{\circ}$, and the pulse uncountable. The abdomen was uniformly and greatly distended, and immobile. Laparotomy was at once performed, and a condition of general peritonitis found, some three pints of purulent fluid escaping when the patient was turned on her side. The source of this was evidently the uterus.

In the right cornu, adjoining but not involving the Fallopian tube was an abscess cavity, equal in size to half-a-crown, and about half an inch deep, which had a ragged opening, and contained offensive débris. At the extremity of the right tube was a small pyosalpinx. The right tube was ligatured and removed, and, as it was not possible to close the abscess cavity by sutures, and the patient's condition contra-indicated hysterectomy, the uterus was fixed to the abdominal wound, after an opening had been made into the posterior cul-de-sac. Five pints of saline solution were injected under the breasts, but the patient never rallied, and died six hours after the operation.

Post mortem, the uterus was found to be full of placental substance and membranes. Cultures from both the uterus and the peritoneal pus (obtained during life) showed gonococci and streptococci.

Case vr. L.B., aged 27, multipara, was confined on March 2nd, 1906, a medical man being present at the time. The labour lasted for four hours only, and instrumental interference was not required.

On March 8th the patient was taken suddenly ill, with shivering and pyrexia; she was seen by the certifying practitioner, who reported the existence of rigors. These were repeated daily until March 12th, and the pyrexia continued until March 15th. During this period there is also a history of sweating, abdominal pain, diarrhœa and daily vomiting.

She was seen daily by the medical man, who prescribed vaginal 
injections, and gave one intra-uterine douche only, viz., on March 8th. The patient was sent to hospital on March 15th.

On admission she appeared to be moribund; there was intense septicæmia, with much prostration. The temperature was $103^{\circ}$, and the pulse could not be counted. There was general abdominal distension, with diminished respiratory movement, and a large mass was easily felt in the right iliac fossa. Laparotomy was at once performed.

On opening the abdomen, the whole of the cavity was seen to be full of pus; the intestinal peritoneum was much inflamed; the intestines themselves were distended and covered everywhere with masses of lymph. Apparently shut off from the general cavity by light adhesions was a large tubo-ovarian abscess on the right side, and the right Fallopian tube was also distended to the size of a sausage with fluid which was subsequently seen to be purulent.

No attempt was made to cleanse the peritoneal cavity. An opening was made into the vagina, behind the uterus, sufficiently free to drain both the abscess cavity and the general peritoneal sac. The pyosalpinx was rapidly ligatured and removed; in this procedure the right broad ligament was seen to be greatly thickened, but was not fluctuating. Drainage tubes were inserted into the vaginal opening and through the lower angle of the abdominal wound, which was then sewn up.

The temperature fell to normal on the third day, and recovery was subsequently uneventful, the patient being discharged well and strong on the 21st May, with the abdominal wound soundly healed.

Cultures from the peritoneal pus showed streptococci only.

CaSE vir. M.P., multipara, aged 23, was admitted to Monsall Hospital on April 11th, 1906.

She had been confined on March 23rd, only a midwife being present. The labour is said to have lasted for two hours only, and to have been followed by considerable hæmorrhage. She was apparently well until April 3rd, when she had a rigor. A medical man saw her the next day for the first time, and he found a temperature of $100^{\circ}$. On April 8th diarrhoea and abdominal pain were present. No local treatment of any kind was adopted until her removal to hospital.

On admission, her condition was extremely grave, but apparently not hopeless. The temperature was $102^{\circ}$, and the pulse-rate 184 . The abdomen was uniformly distended and immobile. There was shifting dulness in each flank.

Laparotomy was at once performed, and a large quantity of turbid fluid evacuated by turning the patient on to her side. The intestines were distended and everywhere coated with masses of lymph. The uterus was enlarged and soft, with pus exuding from 
each Fallopian tube. The right ovary was enlarged and evidently fluctuating. This, together with the right tube, was removed. The patient was then placed in the lithotomy position, and the uterus was curetted with a large sharp curette, and much placental tissue removed. The raw surface was swabbed with undiluted izal fluid, and the cavity packed with izal gauze. A free opening was then made into the peritoneal cavity through the posterior cul-de-sac. Large rubber and gauze drains were then inserted through the abdominal and vaginal wounds; no attempt was made to flush or cleanse the peritoneal cavity.

The patient was distinctly better the next day. She subsequently made an uninterrupted recovery, and was discharged well and strong on June 28th.

A film preparation from the abdominal pus showed streptococci only. The leucocyte count in this case before operation was 27,000 .

Case virr. M.C., multipara, aged 32, was admitted to Monsall Hospital on June 21st, 1906.

This history is, that she had been infected before delivery, and that craniotomy was subsequently required on account of contracted pelvis. This operation was performed on June 19th, the date of the onset of pyrexia being June 18th.

I saw her on admission, in consultation with Sir William Sinclair, who had himself also seen the case some few hours previously. There was no doubt in his mind or my own that the patient was in extremis, and that recovery seemed scarcely possible. The abdomen was greatly distended and motionless; the temperature $102.8^{\circ}$, and the pulse-rate 196. Respiration was gasping in character; the lips were blue; the face was pale, and there was profuse perspiration. It was agreed, however, that an operation should be attempted.

Accordingly, I opened the abdomen through a small median incision in the first instance; a large quantity of turbid fluid gushed out, followed by a coil of intestine, which was distended, its surface being injected and covered with patches of sticky lymph. A drainage tube was inserted, and this wound sewn up. The patient was then placed in the lithotomy position, and the uterus curetted with a sharp curette, swabbed with a 1 in 500 solution of biniodide of mercury, and packed with mercurial gauze.

A free opening was then made into the posterior cul-de-sac, and the abdominal cavity flushed out through the tube in the abdominal incision until the fluid returning through the vaginal opening was quite clear.

100 c.c. of a polyvalent antistreptococcic serum, prepared from five puerperal cultures only, were given subcutaneously in six pints of normal saline solution. The patient rallied well, and on the next day her condition was greatly changed for the better. 
The improvement was maintained, and, with the exception of a pelvic abscess-which was opened by Dr. A. W. W. Lea, who kindly saw the case during my absence on holiday-recovery was uneventful. The patient left the hospital well and strong on September 3rd.

Cultures taken on admission from the blood in the basilic vein showed streptococci, and those from the uterus, streptococci and bacilli coli communes.

The leucocyte count on admission was 16,000 , and 18,000 on the following morning.

CASE IX. B.M., aged 23, primipara, was confined on October 20th, 1906, a medical man being in attendance. Details of the labour were not furnished, but there is said to have been a rise of temperature for the first time on October 23rd. The patient stated that vaginal injections were given daily by a midwife.

No history of treatment was obtainable from the medical man, but it is probable, from the nature of the injuries to the cervix, that the forceps was applied during delivery. The labour was said by the patient to have been of short duration.

She was admitted to Monsall Hospital on October 25th. The general condition was fair, and the colour good; the temperature was 104, and the pulse-rate 140 ; there were frequent rigors. The uterus could be felt just below the umbilicus, and there was very little abdominal distension.

On further examination, there was seen to be a central laceration of the perineum down to, but not into the rectum. The cervix was torn diagonally into the vagina, and the lacerated surfaces were covered with grey sloughs. The uterus contained fragments of placenta and some putrid clot.

The uterine wall was well scraped with a sharp curette, and the raw surface swabbed with undiluted izal, the cavity being subsequently packed with izal gauze.

The rigors were repeated on the next day, but on October 27 th an improvement set in, and was maintained until November 5th, when an attack of parametritis supervened. The uterus was now found to be fixed, but there was no sign of suppuration. From this point the temperature continued high and remittent, but the general condition was fair and the appetite unimpaired; there was but little pain, and no sign of peritonitis.

On November 23rd the patient was taken suddenly ill with shivering, abdominal pain, and collapse. The abdomen rapidly became distended, and did not move well with respiration. A provisional diagnosis of commencing general peritonitis was made, and laparotomy was performed immediately.

On opening the abdomen, a large excess of peritoneal fluid was seen, which was turbid, but not offensive. There were no adhesions 
anywhere; the intestines were distended and covered here and there with small portions of sticky lymph. In the uterine wall, adjoining, but not involving the insertion of the left tube, was an abscess cavity, equal to the size of a shilling, which had evidently only just burst through a small opening, there being no signs of local adhesion. There was also a large cellulitic mass in the parametrium, but no sign of suppuration there.

The abscess cavity was very thoroughly scraped, and swabbed with pure izal, which was subsequently washed away with saline solution. The left tube was ligatured and removed close to the uterine cornu, and the top of the left broad ligament, thus set free, was folded over the site of the abscess, and secured by a double row of Lembert's catgut sutures. The abdomen was then closed without drainage.

No attempt was made to cleanse the general peritoneal surface either by washing or sponging.

Recovery was uneventful; the wound healed well, and the temperature fell to normal 14 days later, by which date the greater part of the parametric inflammation had subsided. The patient left the hospital well and strong, with a movable uterus, on January 31st, 1907.

Cultures taken from the uterus on admission showed streptococci and a bacillus of the coli group. The blood was sterile.

Case x. C.R., aged 34, multipara, was admitted to Monsall Hospital on November 22nd, 1906. She had been confined on November 17 th, the labour being easy and of short duration. Neither a medical man nor a midwife was present at the time of delivery, but the latter arrived in time to "extract" the placenta.

On November 19th, she was seen by the certifying practitioner for the first time. He found her with a temperature of $103^{\circ}$, and a rigor occurred shortly after his visit; there was some abdominal pain, but apparently no other sign. She was not considered to be seriously ill, but as the temperature remained high the uterus was curetted on November 21st. A blunt curette is said to have been employed, and some placental débris was removed; the endometrium was douched subsequently with a 1 in 1000 solution of perchloride of mercury, but was not swabbed.

Within a few hours her condition changed for the worse; there was great pain in the lower part of the abdomen, which also became distended, and on the morning of the next day (November 22nd) she was considered to be suffering from general peritonitis. She was then notified for removal to hospital, the hope being expressed that possibly laparotomy might save her life.

When I saw her on admission, she was obviously moribund. The pulse could not be counted, the temperature was $103^{\circ}$ and the 
respiration gasping in character and entirely thoracic in type. The face was pale, the lips were blue, and the skin was covered with perspiration. The extremities were cold.

My first impulse was not to operate, but the patient's husband and the certifying practitioner were both very anxious that the attempt should be made, so I rapidly opened the abdomen forthwith. A condition of general peritonitis was found, there being about three pints of thick pus free in the abdominal cavity; the intestines were everywhere covered with lymph, both broad ligaments were much thickened, and pus was seen to be issuing from the patent end of each Fallopian tube. Drainage having been established into the vagina and in each flank, the abdomen was closed without any toilet of the peritoneum, and the patient was at once put to bed. Saline infusions (three pints of normal salt solution, 10 minims of adrenalin and $100 \mathrm{cc}$. of a polyvalent antistreptococcic serum) were given under the breasts. The patient did not rally to any great extent, and died six hours later. The operation lasted ten minutes.

Post mortem, the uterus was found to be full of large portions of placental tissue, which when removed formed together a mass equal in size to a large orange; they were all easily detachable with the finger. There was no sign of perforation of the uterine wall. Both Fallopian tubes were partially distended with fetid pus.

The pus which had been removed from the peritoneal cavity at the operation was found to contain streptococci only, the film preparation being everywhere crowded with them. No other organisms were grown in the subsequent cultures.

In this case the sudden alteration for the worse which took place after the curetting suggests that the latter procedure was responsible for the fatal termination of the case. The removal of the placental remains was obviously inefficient, and it is probable that the use of the intra-uterine douche at the same time determined the infection of the peritoneum. It will be noted that no attempt was made-other than by this douche-to disinfect the surface left after the application of the curette.

Any lengthy comment on these cases is, I think, unnecessarythey tell their own tale, and they are not sufficiently numerous to justify the establishment or condemnation of any general principle. Certain points, however, suggest themselves for consideration.

In the first place, all the patients were in a critical condition when the operation was performed; many, as will be seen, were regarded as being in a hopeless state. While I am well aware of the risk one runs of dragging surgical procedures in the mire of lay disapproval by operating under such conditions, I must express my conviction that one's first duty is to give the individual patient a chance, however slight it may be, and I do not think that the 
alternative suggestion that a patient with an abdomen full of pus can recover without operation is worth considering.

Secondly, in only one case (No. viii.) was flushing of the peritoneal cavity resorted to. Drawing for the moment on my own experience of other cases, as well as of those recorded here, I am certainly of opinion that, as a rule, it is best not to flush or even sponge the peritoneal cavity at all, but to rely rather on free drainage at the most dependent part, with subsequent propping-up of the patient in the sitting position, and the subcutaneous injection of saline solution with or without antistreptococcic serum and adrenalin; I may say that I almost invariably give calomel on the second or third day. In Case No. viii. I gladly admit that the flushing which was adopted on the suggestion of Sir William Sinclair may be credited with the patient's recovery.

Thirdly, some of these cases certainly seem to show in what light the isolation hospital is regarded by a certain type of practitioner, viz., as a sort of municipal cemetery. In almost every case of puerperal fever admitted to this hospital which has been attended by the certifying practitioner from the onset, there is a history of much valuable time wasted in the administration of small doses of quinine, internally, and daily vaginal douches. The most that is ever attempted in the way of operative treatment is an intra-uterine douche, and I am not sure that this does not often do more harm than good. One cannot cleanse the uterus in puerperal septicæmia by merely playing on its interior with anything; it needs scrubbing. 